
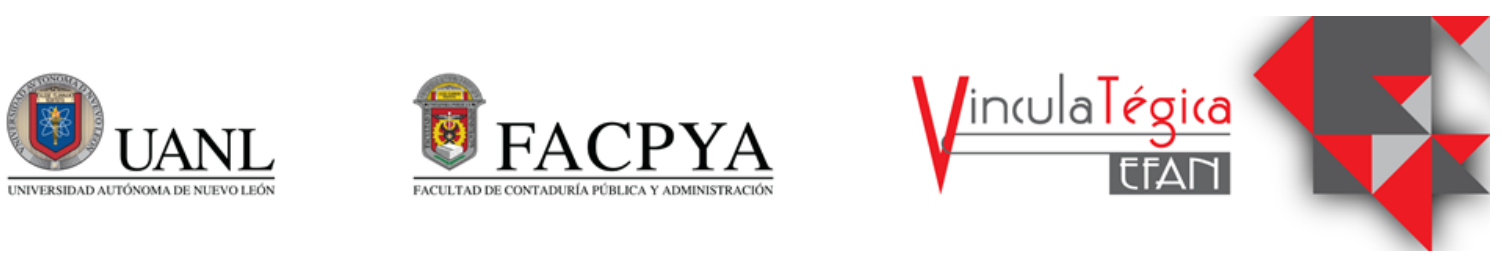

\title{
Retos académicos en la contingencia sanitaria que influyen en la percepción de los estudiantes
}

\author{
María Mayela Terán Cázares ${ }^{1}$, Blanca Nelly Rodríguez Garza² y Ivon Abigail González \\ Garza $^{3}$ \\ ${ }^{1}$ Universidad Autónoma de Nuevo León, Facultad de Contaduría Pública y Administración Monterrey, \\ Nuevo León, mayela.teran@gmail.com Av.Universidad S/N Col. Ciudad Universitaria, (+52) 8183204080 \\ ${ }^{2}$ Universidad Autónoma de Nuevo León, Facultad de Contaduría Pública y Administración Monterrey, Nuevo \\ León, blanca.rodriguezgrz@uanl.edu.mx Av.Universidad S/N Col. Ciudad Universitaria, (+52) 818329 \\ 4080 \\ ${ }^{3}$ Universidad Autónoma de Nuevo León, Facultad de Contaduría Pública y Administración Monterrey, \\ Nuevo León, México, ivonne.gzz.gza@gmail.com Av. Universidad S/N Col. Ciudad Universitaria, (+52) 81
} 26601581

Información del artículo revisado por pares

Fecha de aceptación: junio-2021

Fecha de publicación en línea: diciembre-2021

DOI: https://doi.org/10.29105/vtga7.2-56

\section{Resumen}

El presente estudio tiene como finalidad el identificar la influencia entre los retos académicos y el rendimiento entre los estudiantes de educación superior, para esto se aplicó un instrumento validado con un total de 10 ítems, asimismo este cuenta con una escala Likert con las respuestas enumeradas del 1-5 que van desde el poco de acuerdo hasta totalmente de acuerdo, para la muestra se trabajó con un total de 2913 alumnos que pertenecen a diferentes instituciones de educación de nivel medio y superior del área metropolitana, por otra parte el análisis de resultados se llevó a cabo por medio del estadístico SPSS bajo el modelo de correlación identificando que en un $49.7 \%$ los estudiantes perciben que el modelo hibrido es la oportunidad para continuar con las clases demostrando que directivos, alumnos y profesores deben de trabajar en conjunto para llevar a cabo una formación académica de calidad.

Palabras clave: Retos académicos, rendimiento, estudiantes

JEL: I2, I23

\begin{abstract}
The purpose of this study is to identify the influence between academic challenges and performance among higher education students, for this a validated instrument was applied with a total of 10 items, also this has a Likert scale with responses listed from 1-5 ranging from slightly agree to strongly agree, for the sample was worked with a total of 2913 students belonging to different institutions of education of middle and higher level of the metropolitan area, on the other hand the analysis of results was carried out by means of the SPSS statistic under the correlation model identifying that in $49.7 \%$ of the students perceive that the hybrid model is the opportunity to continue with the classes, demonstrating that directors, students and teachers must work together to carry out a quality academic education.
\end{abstract}

Keywords: Academic Challenges, performance, students

JEL: I2, I23 


\section{INTRODUCCION}

Derivado de la contingencia sanitaria del COVID-19, Ríos (2020) señala que la educación superior tiene como principal reto el fundamentar y dar paso a la modalidad hibrida la cual se caracterice por tener trascendencias y alto nivel de aprovechamiento a nivel virtual como en la presencialidad contemplando como elemento principal la innovación dentro de las prácticas pedagógicas en las aulas.

De esta forma Carreño (2020), enmarca que se requiere de nuevos modelos que se adapten a una nueva formación, proyección y evaluación de actividades, de esta forma se considera a los profesores como actores principales para desarrollar nuevas habilidades y capacidades como profesionistas sin dejar de lado la formación humana y acompañamiento Serano (2020).

Para Shraim \& Crompton (2020) las instituciones educativas deben de trabajar en metodologías innovadoras las cuales incrementen la seguridad y los protocolos definidos sin dejar de lado el desarrollo de nuevas habilidades como seña del aprendizaje significativo.

Asimismo Michotte (2017) indica que el hablar de percepción es centrarse en las actividades que permiten identificar el día a día de los estudiantes al igual se describe su opinión después de tener una experiencia en clases virtuales, la comunidad estudiantil identifica a sus profesores como factores clave en el éxito de sus clases Yusnitua, et. $\mathrm{Al}$ (2021).

Es por ello que el presente estudio se llevó a cabo en diversas instituciones educativas de nivel medio superior y nivel superior dentro del area metropolitana con la finalidad de conocer el impacto de los retos académicos con el rendimiento de los estudiantes dentro de esta nueva modalidad educativa.

\section{MARCO TEORICO}

\subsection{Retos Académicos}

2.1.1 Modalidad Hibrida y rediseño de programas educativos

El regreso a clases presenciales en todos los niveles educativos es de suma importancia y asimismo se considera una prioridad para los directivos de las instituciones de educación superior, Elejalde (2021).

Para ello Rosario (2021) marca como prioridad el estudio detalladamente de las políticas publicas en el tema de educación para formular un rediseño haciendo énfasis en esta nueva realidad educativa y social pos pandemia la cuales se centren en nuevas prácticas, formas de actuar así como nuevas reglas para la convivencia.

Humante en conjunto con demás autores (2019) señalan que para las autoridades educativas es imprescindible tomar posturas que se caractericen por ser concretas y prácticas, facilitando nuevas iniciativas para que el aprendizaje sea generalizado.

A su vez Valero, et. al (2020) señalan que los directivos dentro de su planeación estratégica para el regreso cuentan con responsabilidades y disciplinas para adaptar el ámbito de la modalidad, que transformara los roles tanto de profesores así como de la comunidad estudiantil.

Sin duda alguna el sistema educativo será totalmente diferente durante la pandemia del Coronavirus, haciendo un énfasis dentro del paradigma de la escolarización, esto da paso a la formación de nuevos escenarios donde se involucren modalidades innovadoras considerando las actividades de los docentes y estudiantes para fomentar el aprendizaje.

El retorno a clases representa un área de crecimiento dentro de este cambia completamente los modelos educativos que ya se encontraban establecidos en las escuelas, reflejando una evolución en la productividad, comunicación y pedagogía Henríquez (2020).

De esta forma las instituciones de educación superior deben de reconocer la modalidad hibrida como medio para continuar con las clases ya que para Rosario (2021) esta modalidad "combina la presencialidad con el trabajo en línea tendrá un avance significativo en las trayectorias escolares del estudiantado" (p. 66). 
El objetivo primordial de esta modalidad consiste en no dar paso a sistemas de educativos robustos, si no mas bien recrear $\mathrm{y}$ proporcionar espacios que cuenten con accesos para una educación de calidad con apoyos accesibles (Carreño, 2020).

Por lo que hoy en día las escuelas se encuentran trabajando en la elaboración de protocolos y redefinición de los modelos educativos para continuar con las trayectorias escolares donde los profesores al igual que el personal educativo en su totalidad son actores fundamentales ante la prevención de la contingencia sanitaria (Serrano, 2020).

De esta forma, Sánchez et.al (2020). Identifican 3 tipos de retos las cuales se pueden enfrentar los directivos en el retorno a clases dentro del nivel académico los cuales son:

- La logística: Haciendo referencia a las cuestiones que involucran el manejo del tiempo así como los horarios de clases, asimismo los espacios físicos para impartir las clases son un aspecto para considerar.

- Socioafectivas: Indica todo aquel impacto en las situaciones que tienen que ver con los aspectos emocionales, afectivos y de la salud que viven los docentes como alumnos

- Pedagógicas: Consiste en el reforzamiento de las didácticas de aprendizaje como la creación de nuevas técnicas donde los docentes manejen el manejo de grupos presencial al igual que su evaluación.

\subsubsection{EI Perfil del docente}

De esta forma Soledispa (2020) señala que los docentes necesitan de forma inmediata la formación donde desarrolle nuevas habilidades y capacidades frente a esta nueva realidad, con el principal objetivo de que realizar una adaptación y el ajuste necesario a sus tácticas, y formas de enseñar las cuales se consideran requerimientos educativos.

Asimismo el futuro de los profesores se encuentra incierto la tarea principal consististe en formular nuevas oportunidades para incrementar la interacción en las clases por medio de dinámicas viéndose reflejado en su sentido de confianza y pertenencia (Campbell, 2020).

Por lo que Villafuerte en conjunto con demás autores (2020) identifican que los profesores deben de asumir los siguientes roles como parte de su formación durante la pandemia:

-Orientar en términos académicos: a partir de la enseñanza haciendo que sus estudiantes logren todo aquello que se proponen.

-Poner en practica la escucha activa y la empatía: Básicamente consiste en prestar atención a las demandas de la comunidad estudiantil con la finalidad de que se sientan comprendidos.

- Asesoría institucional: Llevar a cabo diferentes servicios que tengan como finalidad de ayudar a mejorar los procesos de educación dentro del aula y centro educativo

-Motivador: Se encarga de valorar el aprendizaje, guiar a sus alumnos en trabajar en sus objetivos personales.

Shraim \& Crompton (2020) enfatizan como prioridad que los profesores en su regreso participen compartiendo nuevos y mejores conocimientos como el desarrollo de habilidades como parte del proceso de aprendizaje significativo y efectivo.

En base a la literatura revisada se identifican como principales retos académicos:

-La capacitación del docente para las plataformas digitales

-El docente como facilitador durante las clases

profesional

-El retorno a clases como reto

-El modelo hibrido como vialidad para dar continuidad a las clases

\subsection{Rendimiento}

El rendimiento de las instituciones educativas se verá reflejado a la hora de trabajar en la elaboración de diversas y nuevas medidas concentrándose en dar continuidad a la formación de alumnos (Murillo y Duck, 2020).

Longhurst en conjunto con demás autores (2020) indican que las universidades necesitan formar habilidades en donde se 
instruya a profesores y alumnos en esta nueva era, ya sea por medio de la facilitación de capacitaciones cursos o diplomados necesarios para que en conjunto se tomen medidas urgentes.

Para el caso de los estudiantes estar frente a un ambiente totalmente diferente, prosiguiendo con los protocolos de protección formara parte de su proceso de adaptación al igual que la formación de hábitos nuevos dentro del aprendizaje considerando la realidad de los escolares.

Ante esta nueva realidad para la educación superior se muestran cambios como lo enmarca el Secretario General de la OCDE ya que para el 2019 se contaba con 4.5 millones de estudiantes inscritos en un nivel superior con edad oscila entre 18-22 para el 2020 se reportó de un 14\% a $18 \%$ de alumnos inscritos en educación a distancia (Pedró, 2020).

\subsection{La percepción de los estudiantes}

De esta forma Ojeda, et.al (2020) consideran que la percepción del estudiante es un elemento primordial ya que ellos consideran que la formación será mejor si se incorpora las diversas plataformas las cuales son esenciales para enriquecer el conocimiento, asimismo las ideologías de los alumnos contribuyen a la mejora continua del aprendizaje ya que perciben mayores oportunidades de adquirir conocimientos con sentido de innovación.

Igualmente Bermúdez et. al (2020), identifican que gran parte de la comunidad estudiantil tiene una idea de esta nueva modalidad en base a su realidad sociocultural que esta sucediendo ya que en primera instancia señalan que el uso de tecnologías incrementó formulando nuevas practicas educativas.

Sin embargo Sugeng, en conjunto con demás autores (2020) hacen referencia a la percepción de la comunidad estudiantil como elemento esencial de todo proceso educativo identificando en un corto plazo como se sentirán los estudiantes en esta nueva realidad, proyectándose en la organización de las clases al igual que el esparcimiento en las diversas áreas y aulas involucrando las tecnologías como vías de acceso que facilitan el rendimiento

Para que esto se cumpla Rosario (2021) señala que la comunicación será un elemento relevante ya que permitirá tener una mayor interacción entre alumnos y profesores para fomentar espacios donde se permita dialogar, retroalimentar, orientar y autoevaluar el logro de los aprendizajes.

Considerando la literatura revisada se llegó a la pregunta central de la investigación la cual consiste en:

¿Los retos académicos en la contingencia sanitaria influyen en la percepción del estudiante en el rendimiento de las instituciones de educación superior en el modelo hibrido?

Para ello el modelo gráfico se

propone de la siguiente manera en la figura 1

Figura 1Modelo Propuesto

\section{MÉTODO}

El presente estudio es descriptivo

correlacional y explicativo, siendo de forma

transversal considerándolo cuantitativo no

experimental. La muestra se integro por un

total de 2913 estudiantes universitarios que

actualmente se encuentran inscritos en

diferentes instituciones públicas y privadas de educación superior en el área metropolitana.

En primera instancia se valido el instrumento con las variables anteriormente señaladas, en primera instancia se realizó una revisión bibliográfica considerando el protocolo de seguridad de la UNESCO (2021), el cual sirve como una guía práctica y que tiene como objetivo el aportar una serie de medidas sencillas y concretas para que se puedan implementar para adaptar las 
diferentes instituciones y dar continuidad a la educación, asimismo contiene una serie de verificaciones para los directivos y supervisores educativos para llevar a cabo un regreso seguro a las clases.

De esta forma se diseñaron los ítems y de igual forma se adaptaron a un lenguaje propio y entendible para la comunidad estudiantil.

En primera instancia la aplicación del instrumento se llevó a cabo por medio de la prueba piloto que cual consisto de 116 estudiantes, el test consistió de un total de 13 ítems y se utilizó la escala de Likert del 1 al 5 para su respuesta, donde 1 es totalmente en desacuerdo, 2 en desacuerdo, 3 ni de acuerdo ni en desacuerdo, 4 de acuerdo y 5 totalmente de acuerdo. En los datos demográficos recopilados se analizó la carrera, género, edad y universidad de procedencia. Lo cual permitió validar a través del Alpha de Cronbach, los cuales se muestran en la tabla 1:

Tabla 1 Alpha de Cronbach de las variables de estudio

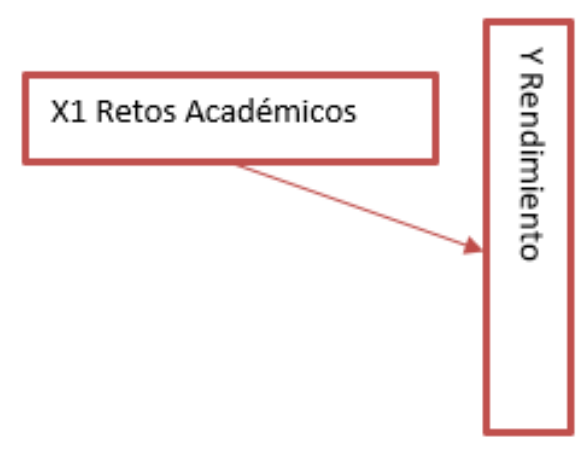

\begin{tabular}{|c|c|c|}
\hline Variable & $\begin{array}{l}\# \quad \text { de } \\
\text { Ítems }\end{array}$ & $\begin{array}{l}\text { Alfa de } \\
\text { Cronbach } \\
\text { Original }\end{array}$ \\
\hline $\begin{array}{l}\text { X1 Retos } \\
\text { académicos }\end{array}$ & 5 & .821 \\
\hline $\begin{array}{l}\text { Y } \\
\text { Rendimiento }\end{array}$ & 5 & .872 \\
\hline
\end{tabular}

\section{RESULTADOS}

\subsection{Modelo}

Los análisis se llevaron a cabo a partir del SPSS V21 y los resultados que se obtuvieron en la regresión lineal múltiple y correlación de las variables independientes:

Retos Académicos

\begin{tabular}{|c|c|c|c|c|c|}
\hline \multicolumn{6}{|c|}{ Resumen del modelob } \\
\hline $\begin{array}{l}\text { Mod } \\
\text { elo }\end{array}$ & $\mathrm{R}$ & $\begin{array}{c}\mathrm{R} \\
\text { cuadra } \\
\text { do }\end{array}$ & $\begin{array}{c}\mathrm{R} \\
\text { cuadrado } \\
\text { corregid } \\
\mathrm{a}\end{array}$ & $\begin{array}{c}\text { Error típ. } \\
\text { de la } \\
\text { estimaci } \\
\text { ón }\end{array}$ & $\begin{array}{l}\text { Durbin- } \\
\text { Watson }\end{array}$ \\
\hline 1 & $.589^{\mathrm{a}}$ & .347 & .347 & .62278 & 1.956 \\
\hline
\end{tabular}

Fuente: SPSS V21

(PROMEDIOACADEMIC), en relación con la variable dependiente Rendimiento (PROMEDIOREND), se describen en la tabla 2. Identificando que el modelo determina la influencia entre la variable dependiente.

Tabla 2 Resumen del Modelo 
En primera instancia se identifica que el valor del Dubrin Watson de 1.956 (ver tabla 1), la cual comprueba la independencia y marca el auto correlación dentro de la regresión, así mismo se identifica que la regresión lineal múltiple arrojo un coeficiente aceptado (r) de .589 por parte del modelo de la investigación al igual que el coeficiente de determinación señala a 0.347 .

\subsection{Análisis de Varianza}

El análisis de varianza ANOVA se caracteriza por comparar las medias de las variables de estudio para definir si existe una relación, dentro del estudio se identifica que es aceptado como se muestra en la tabla 3 , señalando la independencia con un valor inferior al 5\% de la significancia.

Tabla 3 Análisis de Varianza

\begin{tabular}{lcrrrr}
\multicolumn{5}{c}{ ANOVA } \\
\hline Modelo & $\begin{array}{c}\text { Suma de } \\
\text { cuadrados }\end{array}$ & gl & $\begin{array}{c}\text { Media } \\
\text { cuadrat } \\
\text { ica }\end{array}$ & F & Sig \\
\hline $\begin{array}{l}\text { Regre } \\
\text { sión }\end{array}$ & 599.568 & 1 & 599.58 & 1545.856 & $.000^{\mathrm{b}}$ \\
$\begin{array}{l}\text { Resid } \\
\text { uo }\end{array}$ & 1129.433 & 2912 & .388 & \\
\hline Total & 1729.001 & 2913 & & & \\
\hline
\end{tabular}

a. Variable dependiente: PROMEDIOREND

b. Variables predictoras: (Constante), PROMEDIOACADEMIC

Fuente: SPSS V21

\subsection{Correlaciones}

Por su parte las correlaciones señalan el grado de impacto dentro del estudio que los alumnos perciben un impacto de (49.7\%) como el reto que mayor influye es el considerar el modelo hibrido como medio para poder asistir a las clases.

En la tabla \# 4 se puede observar que el reto académico que tiene menor impacto en los estudiantes es el ver este regreso a clases como un reto profesional (21.2\%)

Tabla 4 Correlación entre las variables de estudio 
Fuente: SPSS V21

\section{CONCLUSIONES}

Los estudiantes identifican que dentro de la modalidad hibrida se debe de trabajar con mayor interacción en los lineamientos académicos, para ellos el regreso a clases representan una oportunidad de crecimiento.

La comunidad estudiantil percibe en un $47.9 \%$ que sus docentes deben de estar en constante capacitación en base a nuevos temas que surgen conforme avanza la educación esto con la finalidad de que puedan impartir mejor sus clases las cuales fomenten la participación y colaboración grupal.

Para ello los profesores deben de apoyar constantemente en las actividades, evidencias, tareas y exámenes brindando una mejor atención y servicios, analizándose en un $46.7 \%$ los nuevos roles que los profesores ejercerán durante esta nueva modalidad

Asimismo la comunidad estudiantil identifica en un $21.2 \%$ el retorno a clases como un reto profesional para ellos las clases hibridas significan un modelo para continuar $\mathrm{y}$ adaptando al mismo tiempo la ventaja competitiva de las tecnologías.

De igual forma el estudio sirve como fundamento para formar diversas alternativas para dar una solución a los problemas emergentes que puedan surgir, contribuyendo a realizar futuras investigaciones para el momento que se tenga que regresar

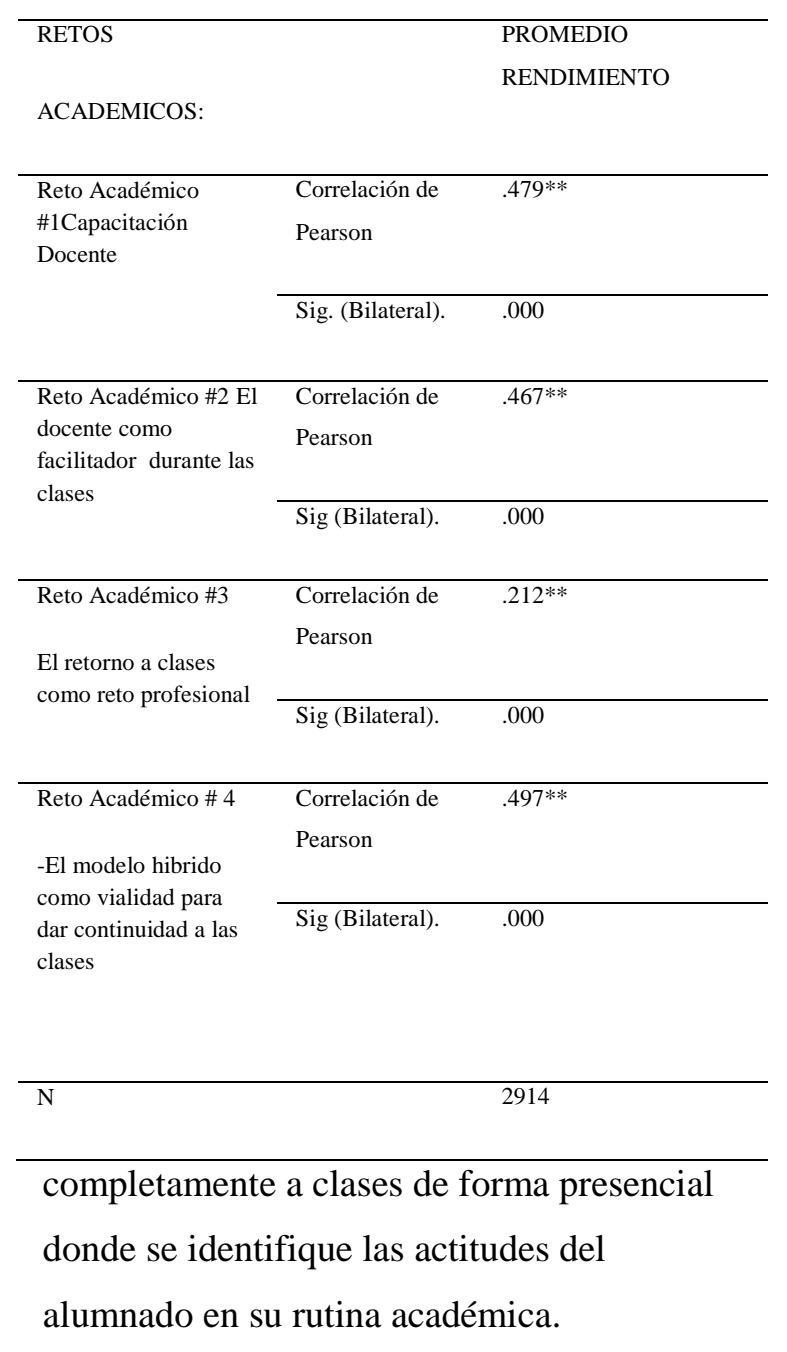




\section{REFERENCIAS BIBLIOGRAFICAS}

Bermúdez, I. J. A., León, E. V., \& Torres, M. J. P. (2020). COVID-19 y Educación Médica: la Virtualidad desde la Perspectiva del Profesor y del Estudiante de Educación Superior. Medicina Interna, 36(3), 116-123.

Campbell, P. (2020) Rethinking professional collaboration and agency in a post-pandemic era, Journal of Professional Capital and Community 5 (34): 337-341 https://doi.org/10.1108/JPCC-06-2020-0033

Carreño, C.I. (2020). Sector educativo. Voces y senderos posibles durante y post Coronavirus Covid-19. Academia https://www.academia.edu/43059307/Sectn or educativo Voces y senderos posibles durante y post Coronavirus Covid 19

Elejalde, R. (2021). La vuelta a clases con el COVID-19. Observatorio Económico, (152), 6-7. https://doi.org/10.11565/oe.vi152.406

Henríquez, H. V., (26 de Abril del 2020). La pandemia un reto a la educación. El mundo.com https://www.elmundo.com/noticia/La-pandemia-un-reto-a-la-educacion/379264

Humante, P., Fernández, J., \& Jiménez, C.(2019). Aulas virtuales en contextos universitarios: percepciones de uso por parte de los estudiantes. Revista Espacios 40(02), 1-17. En: http://www.revistaespacios.com/a19v40n02/a19v40n02p03.pdf

Longhurst, G. J., Stone, D. M., Dulohery, K., Scully, D., Campbell, T., \& Smith, C. F. (2020). Strength, Weakness, Opportunity, Threat (SWOT) Analysis of the Adaptations to Anatomical Education in the United Kingdom and Republic of Ireland in Response to the Covid-19 Pandemic. Anatomical sciences education, 13(3), 301-311. https://doi.org/10.1002/ase.1967

Michotte, A. (2019). The Perception of Causality (1sted). London: Routledge

Murillo, F. Javier, \& Duk, Cynthia. (2020). El Covid-19 y las Brechas Educativas. Revista latinoamericana de educación inclusiva, 14(1), 11-13. http://dx.doi.org/10.4067/S0718$\underline{73782020000100011}$

Pedró, Francesc. (2020). COVID-19 y Educación Superior en América Latina y el Caribe: efectos, impactos y recomendaciones políticas. Fundación Carolina. Junio, Madrid, España.https://www.fundacioncarolina.es/wp-content/ uploads/2020/06/AC-36.-2020.pdf 
Sánchez Mendiola, M., Martínez Hernández, A., Torres Carrasco, R., De Agüero- Servín, M., Hernández- Romo., A., Benavides- Lara, M., Rendon Cázales, V., y Jaimes- Vergara, C., (2020). Retos educativos durante la pandemia de COVID 19: una encuesta a profesores de la UNAM. Revista Digital Universitaria 21(3): DOI: http://doi.org/10.22201/codeic.16076079e.2020.v21n3.a12

Serrano Prato, P. G. (2020). Liderazgo Educativo en tiempos post pandemia: Aportes claves desde una mirada filosófica educativa. Saberes Andantes, 3(7), 48*68. http://saberesandantes.org/index.php/sa/article/view/58

Ríos, J. (09 de Abril del 2020). Después de la pandemia, la educación superior debe transitar a un modelo híbrido. Red Universitaria de Jalisco https://www.udg.mx/es/noticia/despues-de-lapandemia-la-educacion-superior-debe-transitar-un-modelo-hibrido

Rosario- Muñoz, V. (2021). Capitulo 3 Reconfiguración de la educación superior universitaria en México, en tiempos de pandemia por la COVID-19. En Vargas, I. \& Alvarado, M. (Eds), La pandemia de la COVID-19 como oportunidad para repensar la educación superior en México Políticas, prácticas y experiencias (pp.63-85)

Shraim, K., \& Crompton, H. (2020). The Use of Technology to Continue Learning in Palestine Disrupted with COVID-19. Asian Journal of Distance Education, 15(2), 1-20. https://doi.org/10.5281/ZENODO.4292589

Soledispa Cañarte, B. J., Avilés Almeida, P. A., \& Rivera Barberán, G. R. (2020). Desafíos que afrontar para el mundo post pandemia. E-IDEA Journal of Business Sciences, 2(6), 12-21. Recuperado a partir de https://revista.estudioidea.org/ojs/index.php/eidea/article/view/36

Sugeng, E. J., Cock, M., Leonards, E. G., \& Bor, M. V. (2020). Toddler behavior, the home environment, and flame retardant exposure, Chemosphere 252 2-12. https://doi.org/10.1016/j.chemosphere.2020.126588

UNESCO, (2021). Regreso seguro a la escuela: Una guía para la práctica. https://en.unesco.org/sites/default/files/regreso-seguro-a-la-escuela-guia-para-lapractica.pdf

Valero- Cedeño, N., Castillo- Matute, A., Rodriguez-Pincay, R., Padilla- Hidalgo, M., y Cabrera Hernández, M. (2020). Retos de la educación virtual en el proceso enseñanza aprendizaje 
durante la pandemia de COVID-19, Dominio De las Ciencias 6(4): 1201-1220 DOI: https://doi.org/10.23857/pocaip

Villafuerte, J., Cevallos, Y. P., \& Vidal, J. O. B. (2020). Rol de los docentes ante la crisis del Covid-19, una mirada desde el enfoque humano. REFCalE: Revista Electrónica Formación y Calidad Educativa, 8(1), 134-150.

Yusnita, N., Sunaryo, W., y Yulianti, S. (2021). Improving organizational citizenship behaviors (OCB) through strengthening organizational climate and personality Journal of social sciences and humanities 23(1): 19-27

DOI: https://doi.org/10.24198/sosiohumaniora.v23i

$\underline{1.30284}$ 